\title{
Matching Game Based Virtualization in Shared LTE-A Networks
}

\author{
Eftychia Datsika*, Angelos Antonopoulos ${ }^{\dagger}$, Nizar Zorba ${ }^{\S}$, Christos Verikoukis ${ }^{\dagger}$ \\ ${ }^{*}$ IQUADRAT Informatica S. L., Barcelona, Spain \\ ${ }^{\dagger}$ Telecommunications Technological Center of Catalonia, Castelldefels, Spain \\ ${ }^{\S}$ Qatar University, Doha, Qatar \\ Email: edatsika@iquadrat.com, \{aantonopoulos, cveri\}@cttc.es, nizarz@qu.edu.qa
}

\begin{abstract}
Wireless network virtualization enables efficient network sharing in Long Term Evolution Advanced (LTE-A) networks. For the accommodation of ever-increasing user demands, Mobile Virtual Network Operators (MVNOs) can lease and operate virtual resources in network infrastructure that belongs to Mobile Network Operators (MNOs). The coexistence of multiple MVNOs that serve users with different Quality of Service (QoS) requirements and spatial distribution in an LTE-A cell further complicates the arising resource allocation problem. Moreover, the MVNOs aim to satisfy the QoS demands for the maximum possible number of users with the minimum possible cost. This multifaceted context renders centralized optimization approaches for virtual resource allocation in shared LTE-A networks unsuitable. As an alternative, the framework of matching theory, thanks to its distributed nature, can achieve a proper allocation through a stable matching between resources and MVNOs. In this context, we introduce a matching-theoretic formulation for the virtual resource allocation problem and propose a distributed algorithm that properly matches the MVNOs with the available resources. Our simulation results show that the proposed algorithm reaches a stable matching that satisfies the QoS demands for more users in comparison with other approaches.
\end{abstract}

Index Terms-Wireless Network Virtualization, Network Sharing, Matching Theory, LTE-A networks.

\section{INTRODUCTION}

The unprecedented advancement of wireless technologies has introduced a great variety of mobile services, leading to an unparalleled increase of mobile data traffic worldwide. Modern mobile devices offer a plethora of multimedia-based applications with high bandwidth demands, which complicate further the network resources management. Striving to satisfy the end users' Quality-of-Service (QoS) demands, the mobile network operators aim to gain access to new spectrum bands and upgrade the network infrastructure, from base stations to backhaul network components. The Capital (CapEx) and Operation (OpEx) expenditures that stem from the deployment or acquisition of additional network components are key factors to operators' investment strategies.

The inter-operator collaboration seems to alleviate the problem of high network operation cost. The upcoming Long Term Evolution Advanced (LTE-A) technology introduces novel business models, such as network sharing, which allows the pooling and sharing of both infrastructure and licensed spectrum among different operators [1]. Notably, it has been estimated that the cost savings can reach up to $30-40 \%$, proving that network sharing makes economic sense [2]. In the LTE-A multi-operator environment, the high complexity of network management, the variety of services and the tremendous amount of circulating data have motivated the virtualization of wireless network resources. The virtualization allows the abstraction of physical resources, i.e., infrastructure and spectrum, into virtual resource slices (VRSs) and facilitates the network sharing among operators by slicing and isolating network elements [3].

In shared wireless virtualized networks (WVNs), apart from the mobile network operators (MNOs), i.e., the infrastructure and spectrum owners, mobile virtual network operators (MVNOs) with their own mobile customers but without radio access network (RAN) and spectrum ownership may participate in the wireless market [4]. The VRSs may correspond to different evolved NodeB base stations (eNBs) belonging to different MNOs. The MVNOs are interested in accessing the shared physical network and spectrum and manage the VRSs autonomously by applying their own resource allocation and pricing strategies. In order to satisfy the requests of the connected user equipment terminals (UEs), the MVNOs lease and operate VRSs by providing the MNOs (VRS owners) with monetary payment. According to the QoS requirements and the users' spatial distribution, the MVNOs decide about the VRSs they need in each eNB and whether an agreement with the respective MNO-owner is beneficial. They can be associated with multiple MNOs concurrently, selecting the most profitable business agreements (Always-Best-Connected model) [5].

The existence of multiple operators perplexes the VRS allocation in shared WVNs. The MVNOs aim to satisfy the QoS requirements of the maximum possible number of UEs by obtaining a suitable amount of VRSs with the minimum possible cost. Additionally, the users' time-varying wireless channel conditions, QoS demands and spatial distribution in a cell affect the VRS requirements of MVNOs even in shortterm basis. Hence, the availability of VRSs varies according to MVNOs' decisions, as they have to accommodate the incoming requests. The conjunction of the aforementioned factors poses new challenges to the operators regarding the VRS allocation process.

So far, quite a few works have been presented that address the problem of VRS allocation in multiple MVNOs. They 
model the MVNOs as players that compete for physical resources [6], [7], [8] or propose heuristic algorithms to solve multi-objective optimization problems [9]. Although these approaches are quite effective, they require strictly defined utility functions that quantify the operators' preferences and derive solutions in a centralized manner. They also do not consider the coexistence of multiple MNOs that own eNBs in an area, a factor that affects the sharing agreements between operators. Overall, existing approaches do not capture the context that defines the operators' preferences, stressing the need for distributed and self-organizing techniques. Towards this direction, matching theory has recently emerged as a promising solution for complex resource allocation problems of wireless networks [10]. Motivated by the arising issues in VRS allocation, we formulate a matching-theoretic VRS allocation problem, where multiple MVNOs gain access to VRSs of eNBs of multiple MNOs in a shared LTE-A WVN. Next, we propose a distributed algorithm that allocates VRSs to MVNOs, reaching a stable matching where the maximum possible number of MVNOs' mobile customers achieve the requested QoS. The main advantages of the proposed approach are the following:

(i) The MVNOs can declare interest for sets of VRSs in different eNBs owned by different MNOs in a dynamic manner. As the distribution and the UEs' QoS demands may change, the MVNOs can "react" by seeking to be matched with VRSs.

(ii) The participating MVNOs can autonomously rank their preferences over VRSs aiming to serve the maximum possible number of UEs. Thus, a central controller that keeps track of the players' preferences is not required, enabling a flexible VRS allocation.

(iii) The proposed approach is compatible with the LTE-A specification and easily applicable in realistic shared WVNs, as it exploits network management capabilities offered by LTE-A.

The rest of the paper is organized as follows. The considered architecture of shared WVN is described in Section II. In Section III, the problem formulation is presented. The distributed matching-theoretic VRS allocation algorithm is described in Section IV. Simulation results are provided in Section V and conclusions are drawn in Section VI.

\section{Architecture of Shared LTE-A WVN}

We thereupon describe the LTE-A framework for shared LTE-A WVNs and the considered system model.

\section{A. LTE-A framework for network sharing}

In shared LTE-A WVNs, several MNOs own and manage RAN elements, such as eNBs in an area, where MVNOs without any spectrum and infrastructure aim to provide mobile services to their clients (Fig. 1(a)). The eNBs allocate combinations of subcarriers in the time-frequency domain to UEs in the form of Resource Blocks (RBs). The spectrum of each eNB is sliced and shared, thus the VRSs offered to operators in order to accommodate incoming UE requests

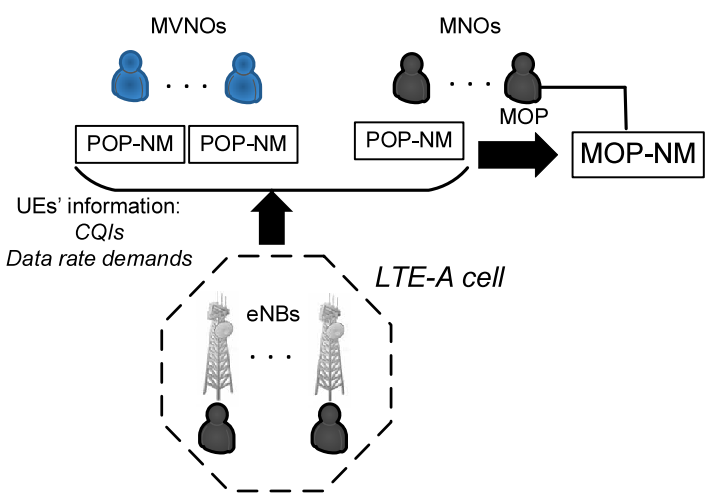

(a) Network sharing in LTE-A

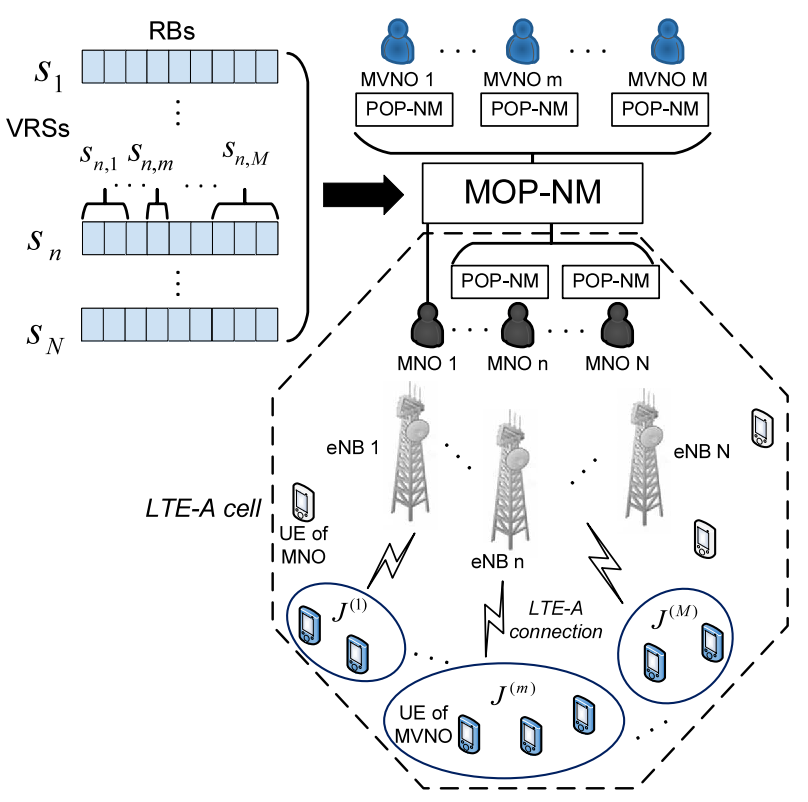

(b) Considered shared LTE-A WNV

Figure 1: Architecture of shared LTE-A WNV

are actually sets of RBs. According to the LTE-A network sharing management architecture, the Master Operator (MOP) controls the shared eNBs using the Master Operator Network Manager (MOP-NM) [11]. One MNO acts as MOP, while the rest of the MNOs and the MVNOs are the Participating Operators (POPs). Each POP uses the Participating Operator Network Manager (POP-NM) to communicate with the MOPNM. When VRSs are allocated to operators, they gain access to the POP-NMs.

The operators should be aware of the UEs' QoS demands and experienced downlink channel conditions in order to decide about the VRSs they need for the accommodation of the users' requests. The eNBs inform the MOP-NM about new UE requests, their downlink channel state and their data rate constraints. The MOP-NM provides this information to POP-NMs. The UEs may receive signals from multiple eNBs 
and estimate the downlink channel quality for each eNB. Each UE can connect to an eNB and report the experienced channel quality by declaring its Channel Quality Indicator (CQI). This determines the modulation and coding scheme (MCS) that can be used for the downlink transmission. The CQIs are available to the MOP-NM and POP-NMs.

\section{B. System model}

We consider the shared LTE-A WVN depicted in Fig. 1(b), where MNOs operate co-located eNBs in a cell. Each MNO owns one eNB $n \in \mathcal{N}$ and licensed spectrum that can be leased to $\mathcal{M}$ MVNOs. A fixed spectrum portion is reserved by MNOs for the requests of their own mobile customers, while the rest can be allocated to MVNOs. The MOP-NM virtualizes the eNBs' resources in a way that $s_{n}$ RBs correspond to eNB $n$. Thus, a set of RBs will be leased to an MVNO $m$, out of which $s_{n, m}$ belong to eNB $n$. Each MVNO requests a set of RBs in each eNB, namely a VRS $s=\left(s_{1, m}, \ldots s_{|\mathcal{N}|, m}\right)$, where each element $s_{n, m} \in\left[0, s_{n}\right]$. The set of available VRSs $s$ is denoted as $\mathcal{S}$. A VRS belongs to exactly one MVNO for a certain time. An MVNO can be associated with multiple RBs.

A set of $\mathcal{J}$ UEs that belong to different MVNOs coexist in the cell. Letting $m$ be an MVNO, we set $\mathcal{J}^{(m)}$ to be the set of UEs of the MVNO. Each UE $j$ imposes rate constraints to the MVNO $m$ it belongs, namely it requests to be served with a minimum downlink data rate $r_{\mathrm{srv}}^{(m, j)}$. A subset of RBs in a VRS $s_{n, m}^{(j)} \leq s_{n, m}$ is allocated to a UE $j$. A UE $j$ reports CQIs for each eNB $n$ from which it receives signal. Given an $\operatorname{MCS}_{n, m}^{(j)}$ and a number of allocated RBs $s_{n, m}^{(j)}$, the estimated downlink data rate of a UE $j$ is:

$$
r_{n}^{(m, j)}=\frac{L\left(\operatorname{MCS}_{n, m}^{(j)}, s_{n, m}^{(j)}\right)}{8 \cdot \mathrm{TTI}},
$$

where the transport block size $L\left(\operatorname{MCS}_{n, m}^{(j)}, s_{n, m}^{(j)}\right)$ can be found in [12] and TTI is the Transmission Time Interval. As the UEs are located in various distances from the eNBs and a fixed transmission power $P_{\mathrm{Tx}}^{(n)}$ is assumed for each eNB $n$, the UEs experience different Signal-to-Noise Ratio (SNR) levels, thus different CQI values are reported. The downlink SNR of each UE $j$ connected to eNB $n$ is estimated as $\operatorname{SNR}_{n}^{(j)}[\mathrm{dB}]=$ $P_{\mathrm{Tx}}^{(n)}-P L_{n}^{(j)}-N_{0}$, where $N_{0}$ is the noise power spectral density $(\mathrm{dBm} / \mathrm{Hz})$ and $P L_{n}^{(j)}$ is the path loss derived by the modified COST231 Hata urban propagation model [13].

\section{MATChING GAME FORMULATION}

In this section, we define the preferences of the participating operators and formulate the VRS allocation problem.

\section{A. Involved operators' preferences}

From the MVNOs' viewpoint, it is important to accommodate as many UE requests as possible by obtaining the lower possible amount of VRSs. Each MVNO aims to obtain a number of VRSs in order to achieve the requested downlink data rates $\sum_{j \in \mathcal{J}^{(m)}} r_{\mathrm{srv}}^{(m, j)}$. For each UE, the MVNO opts for the VRSs belonging to eNBs that can satisfy the UE's rate constraint. The set of UEs of MVNO $m$ that are served, i.e., their minimum rate requirements are satisfied $\left(r_{n}^{(m, j)} \geq r_{\mathrm{srv}}^{(m, j)}\right)$, is denoted as $\mathcal{J}_{\mathrm{srv}}^{(m)} \subseteq \mathcal{J}^{(m)}$. The aim of each MVNO $m$ is to maximize $\left|\mathcal{J}_{\text {srv }}^{(m)}\right|$, namely to minimize the probability that a UE does not achieve the minimum rate defined as Grade of Service (GoS):

$$
G o S_{m}=1-\frac{1}{\left|\mathcal{J}_{m}\right|} \sum_{j \in \mathcal{J}^{(m)}} \sum_{n \in \mathcal{N}}\left[r_{n}^{(m, j)}\left(s_{n, m}^{(j)}\right) \geq r_{\mathrm{srv}}^{(m, j)}\right] \in[0,1] .
$$

From the MNOs' side, the revenue of an owner of eNB $n$ stems from the monetary reward provided by the MVNOs that use the RBs of $n$. In the considered VRS allocation problem, the same price is charged by all MNOs. Therefore, the preferences of the MVNOs over VRSs are determined only by the rates provided to their UEs by the eNBs.

\section{B. Matching-theoretic model}

The MVNOs build their preferences over different VRSs. Each MVNO $m$ has a fixed quota $q_{m}$ that represents the maximum number of VRSs required to support all UEs without violating their data rate requirements. The VRSs preferred by an MVNO may belong to different eNBs and each MVNO may be associated with multiple MNOs. However, a VRS $s$ is used only by one MVNO. Hence, the VRS allocation problem is a many-to-one matching game [14]:

Definition 1. In the VRS market of a shared LTE-A WVN, the MVNO-VRS matching game is defined by two finite sets of players, the set $\mathcal{M}$ of $M V N O$ s and the set $\mathcal{S}$ of available VRSs $s$, and a function $\phi:\{\mathcal{M} \cup \mathcal{S}\} \rightarrow\{\mathcal{M} \cup \mathcal{S}\}$, such that the following conditions are met:

(i) $|\phi(m)| \leq q_{m}, \forall m \in \mathcal{M}$,

(ii) $|\phi(s)|=1, \forall s \in \mathcal{S}$,

(iii) $\forall m, s \in \mathcal{M} \cup \mathcal{S}, m \in \phi(s)$ if and only if $s \in \phi(m)$.

The first condition refers to the fact that MVNOs do not get associated with more VRSs than those required to accommodate their UEs' requests. Let us consider a matching $\phi(m)=\left(s_{1}, m\right), \ldots,\left(s_{K}, m\right)$ of MVNO $m$ with $K$ VRSs $s_{k}$, such that $K<q_{m}$, an MVNO $m$ can obtain $q_{m}-K$ more VRSs. In the set $\mathcal{M} \cup \mathcal{S}$, each MVNO appears as many times as the number of the obtained VRSs. The second condition dictates that each VRS is associated exclusively with one MVNO. The third condition represents the fact that the matching is bilateral, namely a VRS is associated with an MVNO (an MVNO duplicate) if and only if the MVNO obtains this VRS. It is possible that an MVNO or a VRS is not associated with any element of the opposite set. In this case, the matching is still valid, as any "unmatched" elements are associated with themselves.

The outcome of the proposed matching game is a matching of MVNOs and VRSs that allows MVNOs to be associated with a number of VRSs equal or lower than their quota, while each VRS can be matched with one MVNO at most. In this VRS allocation problem, a stable matching must be achieved. For this purpose, the concept of pairwise stability is employed [15]. 
Definition 2. In the VRS market of a shared LTE-A WVN, the MVNO-VRS matching is characterized as pairwise stable if it is not blocked by any player (MVNO or VRS) or any MVNO-VRS pair.

A pairwise stable VRS allocation is the state where there exist no subset of the players (MVNOs or VRSs) that can select sets of partners preferred by all players of the subset, by establishing coalitions only among the players of the subset. For pairwise stability, two conditions have to be met: i) individual rationality and ii) responsiveness.

Out of all possible VRS allocations that may arise during the matching process, only some of them are acceptable by the involved players. The MVNOs accept the allocations that allow them to serve the maximum possible number of UEs. The MNOs are interested in establishing partnerships with the MVNOs until all of their RBs are occupied. The matching that produces acceptable outcomes holds the property of individual rationality [14]:

Definition 3. A matching $\phi$ is individually rational if each VRS and MVNO prefers its current match over being unmatched.

A matching is not individually rational, when either a VRS is not acceptable by an MVNO or an MVNO is not acceptable by a VRS.

The property of responsiveness is related to the way the preferences of MVNOs over VRSs are formed [14]. Regarding the responsive preferences of MVNO $m$ we can define that:

Definition 4. For any set $\mathcal{S}^{\prime} \subset \mathcal{S}$ and any VRS $s$ and $s^{\prime}$ that are not in $\mathcal{S}^{\prime}, M V N O m$ prefers $\mathcal{S}^{\prime} \cup s$ to $\mathcal{S}^{\prime} \cup s^{\prime}$ if and only if $s$ is preferred to $s^{\prime}$, and prefers $\mathcal{S}^{\prime} \cup s$ to $\mathcal{S}^{\prime}$ if and only if $s$ is acceptable.

The responsiveness of the MVNOs' preferences denotes that the MVNOs rank each VRS individually in order to obtain sets of VRSs. Otherwise, they should express preferences over each element of the set of the subsets of acceptable VRSs. An MVNO $m$ always prefers to add an acceptable VRS, until the quota $q_{m}$ is reached and prefers replacing a VRS with a better one, e.g., a VRS that satisfies more UE requests.

\section{Network performance optimization}

The MVNOs aim to maximize the number of users that are served by obtaining exactly the VRSs that satisfy the minimum rate requirement. From the network's perspective, the objective is to minimize GoS for all MVNOs, defined as:

$$
G o S=\frac{|\mathcal{J}|-\sum_{m \in \mathcal{M}}\left|\mathcal{J}_{\text {srv }}^{(m)}\right|}{|\mathcal{J}|} \in[0,1] .
$$

The VRS allocation problem can be formulated as a GoS minimization problem:

$$
\min _{s_{n, m}}\left(1-\frac{1}{|\mathcal{J}|} \sum_{m \in \mathcal{M}} \sum_{j \in \mathcal{J}^{(m)}} \sum_{n \in \mathcal{N}}\left[r_{n}^{(m, j)}\left(s_{n, m}^{(j)}\right) \geq r_{\mathrm{srv}}^{(m, j)}\right]\right),
$$

$$
\begin{aligned}
& \text { s.t. } \quad \text { (a) } \sum_{j \in \mathcal{J}_{\text {srv }}^{(m)}} s_{n, m}^{(j)} \leq s_{n, m}, \forall n \in \mathcal{N}, m \in \mathcal{M}, \\
& \text { (b) } \sum_{n \in \mathcal{N}} s_{n, m} \leq q_{m}, \forall m \in \mathcal{M}
\end{aligned}
$$

The condition (a) refers to the VRS capacity constraints, as each UE cannot use more RBs than those allocated to the MVNO that serves it in a specific eNB. Moreover, the condition (b) dictates that the MVNOs do not get associated with more VRSs than those required to achieve the minimum rate demands of their UEs.

\section{VRS ALLOCATION ALGORITHM}

We propose a distributed VRS allocation algorithm that matches the MVNOs of a shared LTE-A WVN with the available VRSs. The MVNOs create descending order preference lists regarding the VRSs. The algorithm starts when the MVNOs with incoming requests need resources and seek to be matched with the VRSs that are on top of their preference list. The algorithm consists of two phases. The first phase refers to the collection of the required information by the MOP-NM. The MVNOs estimate the rate of their UEs in each eNB and create their sorted preference list. Subsequently, the negotiation phase begins. Each MVNO initially proposes to the VRSs that include the exact number of RBs required to satisfy the minimum rate constraint of all UEs. In case that the requested VRSs are available, the $\mathrm{MNO}(\mathrm{s})$ accept the request and the UEs of the MVNO can be served.

If the proposal of an MVNO to a specific $s$ is rejected, the MVNO is informed about the availability of VRSs through POP-NM. A rejection occurs for either of the following reasons: i) one or more eNBs where the MVNO wishes to obtain VRSs offer fewer RBs than those requested and ii) one or more eNBs have already allocated all of their VRSs. In case (i), the MVNO checks whether the minimum rate constraint can be satisfied for some of the UEs by accepting this VRS. The MVNO's preferences that refer to the eNB(s) that have served the UEs providing a reduced number of RBs are removed by the preference list. If there exist UEs that have not received RBs, the MVNO submits a proposal to the next VRSs in the preference list. In case (ii), the MVNO removes all preferences from the list that refer to the eNB(s) that rejected the proposals. The algorithm terminates when no more proposals are submitted.

Proposition 1. The proposed algorithm converges to a pairwise stable matching after a finite number of steps.

Proof. The number of possible sets of VRSs that can be allocated is finite. At the end of the negotiation phase, all preferred VRSs have been evaluated. Thus, there exist no VRSs that can induce an improvement for any MVNO, i.e., a matching offering more RBs that can serve more UEs. 


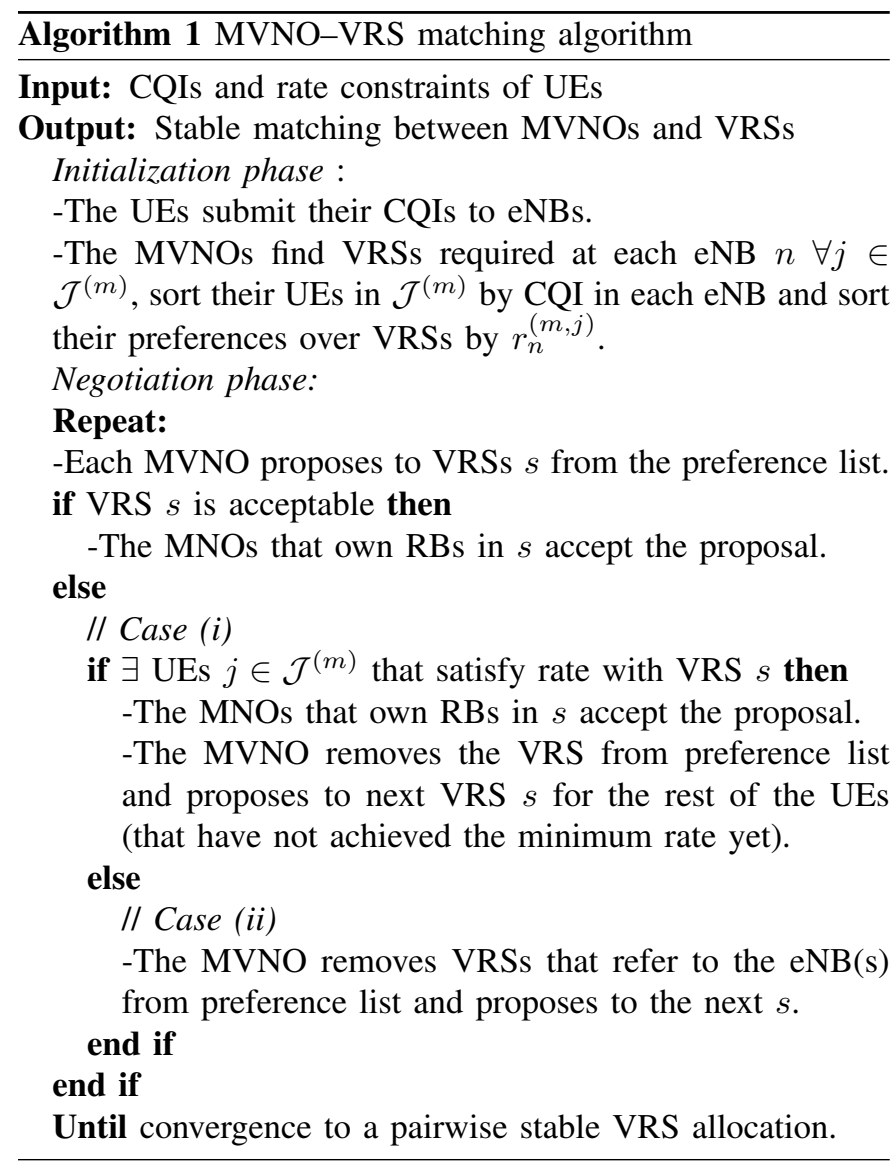

\section{Performance Evaluation}

In this section, we assess the performance of the proposed VRS allocation algorithm in different scenarios using the settings described in Section V-A. The simulation results are discussed in Section V-B.

\section{A. Simulation setup}

We consider the shared LTE-A WVN of Fig. 1, where $|\mathcal{N}|=2$ eNBs belong to two different MNOs, which lease their VRSs to $|\mathcal{M}|=4$ MVNOs. An eNB has a total of 100 RBs that can be used to serve the UEs of MNOs and MVNOs. Each MNO can offer up to $s_{n}=50 \mathrm{RBs}$ to a set of $|\mathcal{J}|=\{20,40,60,80\}$ UEs. A number of $|\mathcal{J}| / 4$ UEs are mobile customers of each MVNO. They are located in random distances from the eNBs and have specific minimum rate demands. We assume two different communication scenarios. In scenario $\mathrm{A}$, a minimum service rate $r_{\mathrm{srv}}^{(m, j)}=64 \mathrm{kbps}$ is required for voice sessions of each UE $j$, while in scenario $\mathrm{B}$, half of the UEs require $r_{\mathrm{srv}}^{(m, j)}=64 \mathrm{kbps}$, while the others need $r_{\mathrm{srv}}^{(m, j)}=256 \mathrm{kbps}$ for data sessions. The simulation parameters are summarized in Table I.

The proposed matching algorithm (denoted as MA) is evaluated in comparison with two VRS allocation approaches: i) the random allocation (RA), which randomly allocates the VRSs to the MVNOs, and the fair allocation (FA) that allocates an equal amount of VRSs to all MVNOs. The performance
Table I: Simulation parameters

\begin{tabular}{||cc||}
\hline \multicolumn{1}{||c||}{ Parameter } & Value \\
\hline \hline Bandwidth per eNB $n \in \mathcal{N}$ & $20 \mathrm{MHz}$ \\
\hline$N_{0}$ & $-174 \mathrm{dBm} / \mathrm{Hz}$ \\
\hline$P_{\mathrm{Tx}}^{(n)} \forall n$ & $46 \mathrm{dBm}$ \\
\hline UE-eNB distance & $500-1500 \mathrm{~m}$ \\
\hline Modulation schemes & QPSK, $16-\mathrm{QAM}, 64-\mathrm{QAM}$ \\
\hline TTI & $1 \mathrm{~ms}$ \\
\hline
\end{tabular}

of all approaches is assessed in terms of GoS and aggregate throughput of the network (AT). For scenario A, we also evaluate the approaches in terms of average cost per UE $(C)$. Given the number of occupied RBs $s_{\mathrm{oc}}^{(n)}$ in eNB $n$ and $p_{n}$ the price charged per RB by the MNO that owns eNB $n, C$ is defined as:

$$
C=\frac{\sum_{n \in \mathcal{N}} s_{\mathrm{oc}}^{(n)} p_{n}}{\sum_{m \in \mathcal{M}}\left|\mathcal{J}_{\mathrm{srv}}^{(m)}\right|} .
$$

\section{B. Simulation results}

In Fig. 2, the GoS performance in both communication scenarios is depicted. The MA achieves lower GoS than the other two, achieving a reduction of $84 \%$ and $88 \%$ in scenario A for $|\mathcal{J}|=20$, comparing to FA and RA, respectively. In scenario B, the GoS with MA is up to 83\% lower comparing to FA and up to $86 \%$ comparing to RA $(|\mathcal{J}|=20)$. The performance improvement induced by the use of MA is attributed to the fact that each MVNO explicitly states the preferred VRSs in each eNB, according to the distribution of the UEs. Fewer RBs remained unused, comparing to the case that FA or RA is employed. With these approaches, VRSs are allocated to MVNOs regardless of their UEs' needs. However, the MVNOs do not need the same amount of RBs in all eNBs, a contingency that is captured by MA. It should be also noted that the GoS increases along with the number of UEs, as more VRSs are required to serve them. Additionally, due to the higher rate demands in scenario B, the GoS is higher, in comparison with the GoS in scenario A for all approaches.

Figure 3 shows the achieved aggregate network throughput AT. For all approaches, AT increases when more UEs exist in the cell, leading to higher VRS demands, thus greater part of the available spectrum is used. The use of MA achieves higher throughput comparing to the other approaches, as a result of the lower GoS MA achieves for all cell congestion levels $(|\mathcal{J}|$ values). The percentage increase of AT with MA compared to FA and RA is up to $26 \%$ and $37 \%$ for scenario $\mathrm{A}(|\mathcal{J}|=60)$, and $28 \%(|\mathcal{J}|=80)$ and $35 \%(|\mathcal{J}|=40)$ for scenario B, respectively. Comparing the AT levels of the two scenarios, we may see that the AT in scenario B is significantly higher than the AT in scenario A. Even though in scenario A the UEs' rate requirements are lower, the available RBs are not always fully utilized. This occurs because the number of RBs allocated to each UE is a discrete value, thus some UEs occupy more RBs than those required to achieve the minimum rate. 


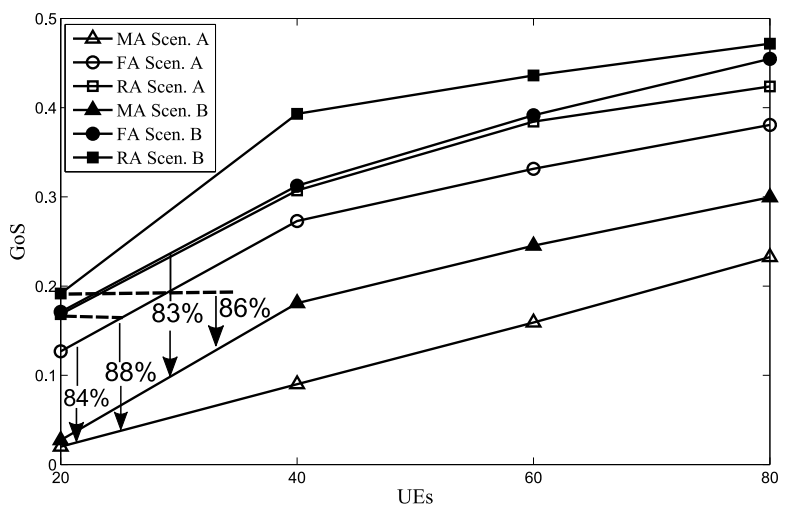

Figure 2: Grade of Service versus $|\mathcal{J}|$

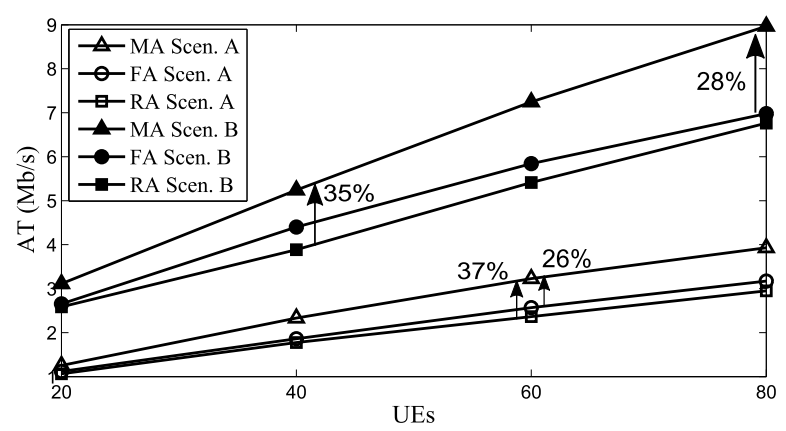

Figure 3: Aggregate network throughput versus $|\mathcal{J}|$

In Fig. 4, the cost per UE in scenario A with $p_{n}=1 \forall n$ is shown. We observe that MA achieves a lower $C$ than the other two approaches, reaching a reduction of $21 \%$ and $33 \%$ comparing to FA and RA, respectively, for $|\mathcal{J}|=80$. MA achieves lower $C$, as more UEs are served, whereas the other approaches do not consider the UEs' distribution. Moreover, $C$ slightly increases when more UEs exist. Even though more RBs are occupied, fewer UEs achieve their rate requirements. From the MNOs' viewpoint, the allocation of more RBs to MVNOs increases the profit, regardless of the GoS levels.

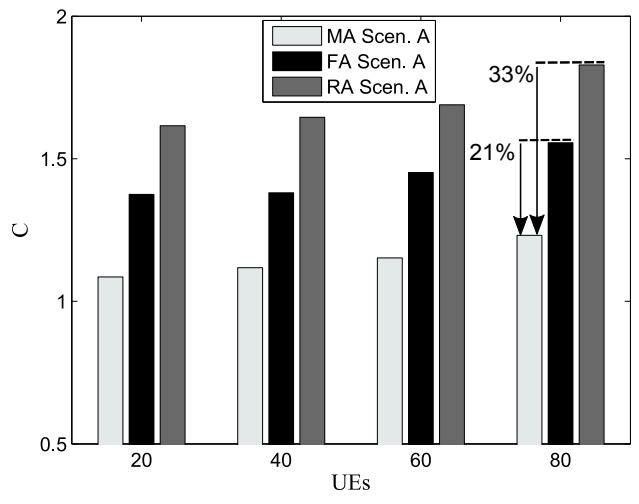

Figure 4: Average cost per UE versus $|\mathcal{J}|$

\section{CONCLUSIONS}

In this paper, a matching game based VRS allocation algorithm has been presented. It exploits the distributed and selforganizing nature of matching theory and achieves a stable matching between MVNOs and VRSs of MNOs, maximizing the probability that UEs are served satisfying their rate constraints. The simulation results have shown that the proposed algorithm performs significantly better than other approaches with respect to the number of served users and the average cost per user, as it enables the MVNOs specify preferences over VRSs autonomously, according to the users distribution and demands. As future work, we plan to investigate the effect of introducing load balancing in the MNOs' preferences.

\section{ACKNOWLEDGEMENT}

This work has been funded by the Research Projects COPCAMS (332913), CellFive (TEC2014-60130-P), AGAUR (2014-SGR-1551) and QUUG-CENG-EE-14 \15-8.

\section{REFERENCES}

[1] OECD Publishing, "Wireless Market Structures and Network Sharing," OECD Digital Economy Papers, , no. 243, 2014.

[2] Body of European Regulators for Electronic Communications, "BERECRSPG report on infrastructure and spectrum sharing in mobile/wireless networks"," BEREC, June 2011.

[3] C. Liang and F.R. Yu, "Wireless Virtualization for Next Generation Mobile Cellular Networks,' IEEE Wireless Communications, vol. 22, no. 1, pp. 61-69, Feb. 2015.

[4] B. Cao, F. He, Y. Li, C. Wang, and W. Lang, "Software Defined Virtual Wireless Network: Framework and Challenges," IEEE Network, vol. 29, no. 4, pp. 6-12, July 2015.

[5] R. Copeland and N. Crespi, "Modelling Multi-MNO Business for MVNOs in their evolution to LTE, VoLTE and Advanced Policy," in 15th International Conference on Intelligence in Next Generation Networks (ICIN), Oct. 2011, pp. 295-300.

[6] B. Liu and H. Tian, "A Bankruptcy Game-Based Resource Allocation Approach among Virtual Mobile Operators," IEEE Communications Letters, vol. 17, no. 7, pp. 1420-1423, July 2013.

[7] G. Zhang, K. Yang, J. Wei, K. Xu, and P. Liu, "Virtual Resource Allocation for Wireless Virtualization Networks using Market Equilibrium Theory," in IEEE Conference on Computer Communications Workshops (INFOCOM WKSHPS), Apr. 2015, pp. 366-371.

[8] F. Fu and U.C. Kozat, "Wireless Network Virtualization as A Sequential Auction Game," in Proceedings IEEE INFOCOM, Mar. 2010, pp. 1-9.

[9] M. Yang, Y. Li, D. Jin, J. Yuan, L. Su, and L. Zeng, "Opportunistic Spectrum Sharing Based Resource Allocation for Wireless Virtualization," in International Conference on Innovative Mobile and Internet Services in Ubiquitous Computing (IMIS), July 2013, pp. 51-58.

[10] Y. Gu, W. Saad, M. Bennis, M. Debbah, and Z. Han, "Matching Theory for Future Wireless Networks: Fundamentals and Applications," IEEE Communications Magazine, vol. 53, no. 5, pp. 52-59, May 2015.

[11] 3rd Generation Partnership Project, "Telecommunication management; Network sharing; Concepts and requirements (3GPP TS 32.130 v. 12.0.0 Rel. 12)," Dec. 2014.

[12] 3rd Generation Partnership Project, "LTE; Evolved Universal Terrestrial Radio Access (E-UTRA); Physical Layer Procedures (3GPP TR 36.213 v. 12.4.0 Rel. 12)," Dec. 2014.

[13] 3rd Generation Partnership Project, "Technical Specification Group Radio Access Network; Spatial channel model for Multiple Input Multiple Output (MIMO) simulations (3GPP TR 25.996 v. 12.0.0 Rel. 12)," Sept. 2014.

[14] A. E. Roth and M. A. O. Sotomayor, Two-sided Matching: A study in Game-Theoretic Modeling and Analysis, Number 18. Cambridge University Press, 1992.

[15] A. E. Roth, "Deferred Acceptance Algorithms: History, Theory, Practice, and Open Questions," International Journal of Game Theory, vol. 36, no. 3-4, pp. 537-569, 2008. 